\title{
Goal specificity and knowledge acquisition in statistics problem solving: Evidence for attentional focus
}

\author{
DAVID L. TRUMPOWER \\ Marshall University, Huntington, West Virginia \\ TIMOTHY E. GOLDSMITH \\ University of New Mexico, Albuquerque, New Mexico \\ and \\ MELISSA J. GUYNN \\ New Mexico State University, Las Cruces, New Mexico
}

\begin{abstract}
Solving training problems with nonspecific goals (NG; i.e., solving for all possible unknown values) often results in better transfer than solving training problems with standard goals (SG; i.e., solving for one particular unknown value). In this study, we evaluated an attentional focus explanation of the goal specificity effect. According to the attentional focus view, solving NG problems causes attention to be directed to local relations among successive problem states, whereas solving SG problems causes attention to be directed to relations between the various problem states and the goal state. Attention to the former is thought to enhance structural knowledge about the problem domain and thus promote transfer. Results supported this view because structurally different transfer problems were solved faster following NG training than following SG training. Moreover, structural knowledge representations revealed more links depicting local relations following NG training and more links to the training goal following SG training. As predicted, these effects were obtained only by domain novices.
\end{abstract}

The goals imposed by a problem-solving task influence what is learned during problem solution. Sweller and Levine (1982) were among the first to show that nonspecific goals (NG; e.g., solving for as many unknowns as possible) benefit learning relative to specific goals (SG; e.g., solving for specific unknowns), a finding that is called the goal specificity effect. As a demonstration of the goal specificity effect, Sweller, Mawer, and Ward (1983) had participants solve multistep transformation problems in the domain of physics (Experiments 2 and 3 ). Half of the participants were given problems with specific goals (e.g., "In $18 \mathrm{sec}$ a racing car can start from rest and travel $305.1 \mathrm{~m}$. What speed will it reach?"), whereas the other half were given problems with nonspecific goals (e.g., "In $18 \mathrm{sec}$ a racing car can start from rest and travel $305.1 \mathrm{~m}$. Calculate the value of as many variables as you can."). Participants given problems with nonspecific goals showed more expert-like performance

Preliminary analyses of the undergraduate data were reported at the 24th Annual Conference of the Cognitive Science Society, held August 7-10, 2002, at George Mason University in Fairfax, Virginia. Undergraduate data were collected while D.L.T. was at the University of New Mexico. We thank Molly Below for her assistance in collection of these data. Correspondence should be addressed to D. L. Trumpower, Department of Psychology, Harris Hall 328, One John Marshall Drive, Marshall University, Huntington, WV 25755-2672 (e-mail: trumpower@marshall.edu). than did those given problems with specific goals. Nonspecific goals led to fewer errors, and to use of forwardworking strategies, which have been shown to be characteristic of experts (Larkin, McDermott, Simon, \& Simon, 1980). Since then, the goal specificity effect has been shown with geometry (Ayres, 1993; Sweller et al., 1983, Experiments 4-7), trigonometry (Owen \& Sweller, 1985; Sweller, 1988), and several more complex, dynamic tasks (e.g., Burns \& Vollmeyer, 2002; Geddes \& Stevenson, 1997; Miller, Lehman, \& Koedinger, 1999; Vollmeyer, Burns, \& Holyoak, 1996). The aim of this article is to examine the processes underlying the goal specificity effect and the generality of knowledge acquired as a result of training with nonspecific versus specific goals. We first introduce two related explanations of the goal specificity effect, and we then describe the different types of knowledge that are presumed to result from specific versus nonspecific goal training.

In the course of problem solving, individuals are assumed to learn schematic knowledge about the problem domain, or at least to learn about the particular type of problem being solved. By schematic knowledge, we mean either general knowledge of the relationships between important domain concepts, as exemplified in high-level principles, or specific knowledge of particular problem types and the appropriate equations needed to solve them. More will be said later about the generality 
of the knowledge acquired in the course of problem solving. In either the general or more specific sense, schematic knowledge is believed to underlie expertise (e.g., Chi, Feltovich, \& Glaser, 1981) and to allow relatively quick and error-free solutions. Consider the physics problem given earlier. Individuals solving this type of problem may learn the relationships between time, distance, and final velocity, or they may learn to recognize this problem type and what equations to apply in order to solve it (i.e., given time and distance, and asked to solve for final speed, apply the equations distance $=$ average velocity $\times$ time and then average velocity $=$ final velocity / 2 ). However, not all problem-solving instances will result in schema learning because schema acquisition is not automatic. The goal specificity effect suggests that a problem's goal can determine the extent of schema learning.

The initial explanation of the goal specificity effect came from cognitive load theory (CLT; Sweller, 1988). CLT posits that specific goal problems focus attention on differences between the current problem state and the goal state, thus encouraging use of a means-ends analytic strategy. Means-ends analysis involves reducing differences between the current state and the goal state by applying legal operators (e.g., equations) until the goal state is achieved (Simon \& Simon, 1978). Although means-ends analysis is a very effective problem-solving strategy, it is also cognitively demanding. Problem solvers who use a means-ends strategy must maintain information regarding "the current problem state, the goal state, the relation between the current problem state and the goal state, the relations between problem-solving operators and, lastly, if subgoals have been used, a goal stack" in working memory (Sweller, 1988, p. 261). Thus, if much of the limited cognitive resources are devoted to using the means-ends strategy, few resources are available for more general learning (i.e., schema acquisition). Nonspecific goal problems, on the other hand, avoid the possibility of using a means-ends strategy because the ultimate goal state is not specified. Rather, nonspecific goal problems encourage a forward solution strategy in which current problem states are compared with possible operators until an immediately applicable operator is found. This process continues until no further applicable operators are found. Whereas means-ends analysis requires that the current state, the goal state, subgoal states, and operators be kept in working memory, a forward solution strategy requires only that the current state and possible operators be kept in working memory. Thus, nonspecific goal problems encourage the less resource-demanding forward solution strategy, thereby freeing up resources that can be used for schema learning.

Sweller (1988) developed a computer model that solved specific and nonspecific goal kinematics problems in order to provide support for the cognitive load explanation. The model was comprised of one set of productions (i.e., if-then condition-action pairs) for solving specific goal problems using means-ends analysis, a second set of productions for solving nonspecific goal problems using a forward solution strategy, and a working memory that held lists of equations, known variables, unknown variables, and the goal (for specific goal problems only). The model solved problems by attempting to match elements in working memory with the conditions of productions. If a condition was satisfied, its production fired. The number of productions that needed to fire in order to solve a problem and the number of elements in working memory that needed to be matched in order to fire the necessary productions were taken as indicators of cognitive load. The model did indeed incur a greater cognitive load when solving specific goal problems than when solving nonspecific goal problems, thereby providing support for CLT. CLT has since been used to describe several findings in the instructional science literature, including the worked example effect (Sweller \& Cooper, 1985; Tuovinen \& Sweller, 1999) and split attention and redundancy effects in multimedia learning (Kalyuga, Chandler, \& Sweller, 2000).

More recently, some of the limits of CLT have been revealed. Specifically, van Merriënboer, Schuurman, de Croock, and Paas (2002) have shown that although some manipulations that reduce cognitive load benefit performance (partially solved vs. conventional problems, Experiment 1), others do not (high vs. low contextual interference, Experiment 2). Similarly, learning conditions that are subjectively viewed as difficult by learners can often lead to slower acquisition but facilitate transfer and retention relative to learning conditions that are viewed as easy and lead to faster acquisition (for a review, see Bjork, 1999). For example, reducing the amount of feedback during training has been shown to slow acquisition but to facilitate transfer in concept formation (Schroth, 1997). If it is assumed that providing feedback lowers cognitive load (which seems like a reasonable assumption because cognitive load researchers have argued that partially solved problems carry a lower cognitive load than do conventional problems, and because participants typically view feedback as making a task easier), these findings appear incongruous with CLT's claim that decreasing cognitive load increases learning.

In response to such potentially incongruous findings, CLT has been modified to allow for both extraneous cognitive load (which arises when attention is focused on processes irrelevant to learning) and germane cognitive load (which arises when attention is focused on processes relevant to learning; Valcke, 2002; van Merriënboer et al., 2002). Thus, focus has shifted to include attentional mechanisms, such that manipulations that focus attention (or focus working memory resources) on extraneous processes are hypothesized to increase load and to impair learning, whereas manipulations that focus attention (or focus working memory resources) on germane processes are expected to increase load and to facilitate learning. Unfortunately, because there are currently no independent measures of extraneous and germane cognitive load, it is impossible to predict the net effect on learning based solely on cognitive load considerations. Therefore, we believe that a more complete understanding of the goal specificity effect can be obtained 
by examining the attentional processes that occur during training with specific and nonspecific goal problems. This approach may help to inform CLT by providing a means to distinguish germane and extraneous cognitive load. Specifically, germane cognitive load may best be defined as focusing attention on aspects of a task that are relevant for successful transfer, and extraneous cognitive load may best be defined as focusing attention on other, transfer-irrelevant aspects of the task. To the extent that this is the case, the technique we use to assess attentional focus in this study can be viewed as a measure of extraneous versus germane cognitive load.

Consistent with the idea that attentional processes can help to explain the goal specificity effect, Miller et al. (1999) proposed an explanation based on "goal-directed knowledge transfer." By goal-directed knowledge transfer, Miller et al. meant that the effects of any pedagogical manipulation can be explained in terms of the specific knowledge acquired during training and the specific knowledge required for successful transfer. In their study, participants were trained to interact with a physics microworld called electric field hockey (EFH) and were later tested for transfer. In EFH, participants place electrical charges in a field in order to maneuver an electrically charged particle, or puck, into a hockey net. The pedagogical objective was to teach users about the relationships between concepts such as force and distance of electrical charges. Participants trained with no goal, in which no hockey net was present and they simply maneuvered the puck around the field, showed better transfer than did those trained with a standard specific goal of getting the puck into a net. However, a third group trained with a more specific goal of trying to keep the puck on a specific path into a net performed as well as did the nogoal group. Miller et al. suggested that the no-goal and the more specific goal conditions worked by focusing attention on the placement of the electric charges and on their immediate effect on the puck across its entire trajectory. This was in contrast to the standard goal condition that focused attention on the difference between the goal and where the puck ended up (i.e., the end of its trajectory). By attending to the effect of the electric charges across the entire trajectory, participants learned the correct relationship between distance and force. When attention was restricted to differences between the end of the trajectory and the goal, however, participants did not attend to the initial effect of the charge, and the relationship between distance and force was obscured. Attending to the distal relation between the initial placement of the electrical charges and how far the puck ends up from the goal can be considered pedagogically irrelevant, therefore demanding extraneous cognitive load, whereas attending to the more immediate, or local, relations between the electrical charges and the entire trajectory of the puck can be considered pedagogically relevant, therefore demanding germane cognitive load.

More generally, an attentional focus view suggests that a nonspecific goal (or several highly specific goals, as in the more specific condition of Miller et al. [1999]) fosters learning of local relations between successive problem states by focusing attention on the immediate effect of operators. A single standard goal encourages learning of more distal relations between current states and the goal state, at the expense of learning local relations. From here on, we will refer to problems that have a single specific goal (e.g., "What is the final velocity?" or "Solve for angle ABC") as standard goal problems since these are the type of problems often found in textbooks, homework assignments, and exams. Standard goals encourage learning of distal relations by focusing attention on how an operator moves one closer to or further from the goal, and not focusing attention on how the operator causes a change from the previous state. Learning how operators move one closer to or further from the goal may be sufficient when problems have identical goals, but knowledge of local relations allows transfer to problems with novel goals. In the present study, we directly test predictions of this attentional focus view.

A separate but closely related issue regarding the goal specificity effect involves the generality of what is learned. Most descriptions of the effect propose that training with nonspecific goal problems leads to enhanced schema acquisition. As evidence, students trained with nonspecific goal problems have repeatedly shown better performance on transfer problems than did those trained with standard goal problems (Ayres, 1993; Bobis, Sweller, \& Cooper, 1994; Miller et al., 1999; Owen \& Sweller, 1985; Sweller, 1988; Sweller et al., 1983; Vollmeyer et al., 1996). However, most studies of the goal specificity effect have restricted their dependent measures to transfer problems that are structurally identical to training problems. For example, Sweller et al. (1983, Experiment 3) asked participants to solve eight training problems that gave values for final velocity and time (and to solve for distance in the standard goal condition). Participants were then tested on two transfer problems that gave values for final velocity and time and asked to solve for distance. Participants trained with nonspecific goal problems solved the two test problems in significantly fewer moves than did those trained with standard goal problems. Although this kind of evidence is consistent with facilitated schema acquisition under nonspecific goal conditions, the knowledge used to solve the transfer problems could be either very narrow or very broad in scope. It is possible that participants trained under nonspecific goal conditions learned what equations to apply in the presence of a specific type of problem, which is defined by its given values. This is precisely what Sweller et al. suggested. This narrow type of knowledge would not be applicable under a different set of given values or problem states. Alternatively, it is possible that participants trained under nonspecific goal conditions learned more general relations among possible problem states (i.e., sets of givens) and moves. Rather than learning to apply certain equations to find distance when given values for final velocity and time, participants might actually have learned the relations between final velocity, time, and distance, such that knowing any two would allow calculation of the third. This type of 
knowledge is broader in the sense that it can be applied to multiple problem types.

Owen and Sweller (1985, Experiment 3) tested the effect of goal specificity on transfer problems that were structurally different from training problems in the domain of trigonometry. They found that participants trained with nonspecific goal problems made fewer errors when solving structurally different transfer problems than did those trained with standard goal problems. This, they concluded, showed that reducing the specificity of training goals facilitates the acquisition of general trigonometric principles. Unfortunately, participants in the nonspecific goal condition were allowed to solve for statistically significantly more unknown values during training than were those in the standard goal condition (a mean of 4.9 and 10.8 unknown values, respectively). Thus, the effect may have been due to differences in the amount of practice rather than to differences in goal specificity. Therefore, the question of the generality of acquired knowledge in demonstrations of the goal specificity effect is still an open question. In the present study, participants in standard and nonspecific goal conditions were restricted to solving for the same number of unknown values, thus controlling for the amount of practice in evaluating the effects of goal specificity.

An attentional focus explanation of the goal specificity effect predicts the acquisition of more general knowledge under nonspecific goal conditions. Under standard goal conditions, attention is focused on a particular goal and on how to achieve that goal at each step of the problem-solving process. Thus, one might be expected to learn how other variables (i.e., givens and knowns) are related to the goal, but not how those other variables are related to one another. Under nonspecific goal conditions, attention is focused on the current problem state and possible moves. Here, one is expected to learn the relationships between the known variables at each current state and those immediately derivable to achieve the next problem state. As such, reduced goal specificity leads to the acquisition of a variety of local relations rather than to relations with one specific goal. Thus, knowledge acquired with standard goal specificity is likely to be tied to problems of a specific goal structure, whereas knowledge attained with reduced goal specificity should be more generally adaptable to problems with different structures. To test this idea, we included in the present study both transfer problems that were structurally identical and transfer problems that were structurally different than those used during training.

We also examined the role of expertise on the goal specificity effect. Assuming that more expert problem solvers already possess knowledge of a domain's local structural relations, focusing attention on those relationships should not benefit any further learning. Also, more expert problem solvers should already possess schemas that eliminate the need to use cognitively demanding strategies, even on standard goal problems. Thus, more expert problem solvers are expected to show no benefit from nonspecific goal problems. Similar expertise by training manipulation interactions have been found with the redundancy effect (Kalyuga et al., 2000), worked examples (Tuovinen \& Sweller, 1999), and problem fading (Renkl, 2002).

\section{SUMMARY AND OVERVIEW}

The aim of the present study was threefold. First, we tested specific predictions made by an attentional focus explanation of the goal specificity effect regarding the kind of relational information attended to and subsequently learned. Second, we examined the generality of knowledge acquired under training with standard and nonspecific goal problems. Third, we considered the influence of expertise on the goal specificity effect.

To accomplish these aims, a group of students with no previous experience with behavioral statistics and a group that had previously completed at least one advanced course in statistics solved three training problems from the domain of one-way analysis of variance (ANOVA) that required solving for either specific standard goals (e.g., "Solve for the between-groups sum of squares") or nonspecific goals (e.g., "Solve for as many unknowns as possible"). Afterward, participants' structural knowledge was assessed. Structural knowledge refers to knowledge of the interrelationships among important domain concepts (Goldsmith, Johnson, \& Acton, 1991). In order to assess their structural knowledge, participants were asked to rate the relatedness of pairs of statistics concepts on a 5-point Likert-type scale. These data were then submitted to the Pathfinder scaling algorithm to generate a network representation of each participant's structural knowledge of the domain. These network representations consist of concept nodes with links between more related concepts. More specifically, the Pathfinder algorithm treats relatedness ratings as proximities and searches for the shortest "path" between two concepts. If the shortest path is a direct one, a link between the two concepts is included in the network. If the shortest path is not a direct one, no link is included (for a more thorough review of Pathfinder networks, see Schvaneveldt, 1990). The quality of one's network can be assessed in various ways - for example, comparing it with a domain expert's network - and has been shown to be a good indicator of general domain knowledge (Goldsmith et al., 1991).

According to an attentional focus view, participants in the standard goal (SG) condition were expected to focus attention on the goal concept (between-groups sum of squares) and the knowns at each problem state. Therefore, SG participants should have more links with the goal concept in their derived knowledge networks than should nonspecific goal (NG) participants. Participants in the NG condition were expected to focus attention on the local relations necessary to solve for an unknown at each new problem state. Because these relations are depicted in the equations used to solve the problems, NG participants should have more of these types of links in their knowl- 
edge networks than should SG participants. These differences between the NG and SG conditions should only arise, however, for the less expert participants. Those with previous statistics experience should already possess knowledge of the links depicted in basic statistics equations and, thus, should not benefit from NG training.

Participants were also asked to solve several transfer problems, half of which were structurally identical to the training problems and half of which were structurally different. Because of the different knowledge expected to be acquired by participants in the SG and NG conditions (goal-specific vs. more widely applicable local relations, respectively), participants in the SG condition were predicted to be more impaired than participants in the NG condition when attempting to solve the structurally different transfer problems. Again, we predicted this difference only for the less expert participants.

\section{Method}

Participants. The participants were 14 undergraduate students enrolled in an introductory psychology course at the University of New Mexico who had not previously completed a college-level statistics course, and 22 graduate students enrolled in an advanced quantitative methods course at New Mexico State University. The students participated for partial course credit or for extra credit. Half of the undergraduate students and half of the graduate students were randomly assigned to receive training with SG problems, and the other half received training with NG problems.

Procedure. All participants were tested individually in the presence of an experimenter. The participants first attempted to solve three training problems from the domain of one-way ANOVA. All problems were word problems that could be solved with the following three equations: $d f_{B}=a-1, M S_{B}=S S_{B} / d f_{B}$, and $F=$ $M S_{B} / M S_{W}$, where $a=$ number of groups, $d f_{B}=$ between-groups degrees of freedom, $M S_{B}$ = between-groups mean square, $S S_{B}=$ between-groups sum of squares, $F=F$ ratio, and $M S_{W}=$ withingroups mean square. The three training problems were similar in that they gave values for $a, M S_{W}$, and $F$. The participants in the SG condition were asked to solve for $S S_{B}$ for each training problem, whereas those in the NG condition were asked to solve for as many unknown values as possible. In both conditions, successful solution required first calculating $d f_{B}$ and $M S_{B}$, in either order, and then calculating $S S_{B}$. Thus, the participants in both the SG and NG conditions were required to use all three equations and to solve for the same number of unknowns. During training, the participants were given separate note cards containing each of the three equations needed to solve the problems, and a calculator to perform computations. The participants were allowed $5 \mathrm{~min}$ to solve each problem, during which time the experimenter would immediately notify the participant if he or she made a mistake. The participants were not told the nature of their mistakes, but if they had not solved a problem within the allotted $5 \mathrm{~min}$, the experimenter guided them to the solution. The participants worked out the solution on paper in response to the verbal prompts of the experimenter, who first reiterated the information that was given and requested in the problem, and then pointed out the first error. Then, the prompts to solution were the same for the SG and NG conditions. The experimenter specified what could be computed next, given the known information. After completing a problem, the participants were instructed to go on to the next problem and not to refer back to previous problems.

Following the three training problems, all the participants were shown all 15 pairwise combinations of the six statistics terms in the equations. They were asked to rate the relatedness of the terms in a pair on a 5 -point scale $(1=$ not at all related, $5=$ very related $)$.
During this task, the three equations were not made available to the participants.

Finally, the participants were asked to solve four transfer problems. The first two transfer problems were structurally identical (S-I) to the training problems in that they gave values for $a, M S_{W}$, and $F$. The last two transfer problems were structurally different (S-D), giving values for a different set of variables. All transfer problems, though, could still be solved with the three equations used during training. The participants in both the SG and NG conditions received transfer problems with specific goals. For the S-I problems, the goal was to solve for $S S_{B}$. The goal for one S-D problem was to solve for $M S_{W}$, and the goal for the other was to solve for $a$ (see the Appendix). Again, the participants were given the equations and a calculator, and problem solving proceeded as during training, with 5 min per problem, during which time the experimenter would immediately notify the participant if he or she made a mistake. The participants who failed to solve a test problem in the allowed time, however, were not provided with the solution, as they were during training.

\section{Results}

Separate analyses were performed for the undergraduate and graduate participants. This was done for two reasons. First, we were not concerned with a main effect of expertise. It was clear that graduate participants would perform at a higher level than undergraduate participants. Second, although the graduate and undergraduate participants were subjected to identical experimental procedures, they were recruited from and tested at different universities. A .05 level of significance was used for all tests, unless otherwise reported.

Training. A 2 (goal specificity) $\times 3$ (problem) mixed ANOVA with repeated measures on the second factor was performed on time to solve the three training problems (see Figure 1). For the undergraduate participants, there was a significant main effect of goal specificity $\left[F(1,12)=8.62, M S_{\mathrm{e}}=3,847.05, p<.05, \eta^{2}=.418\right]$, indicating that participants in the NG condition solved training problems faster than did those in the SG condition. There was also a significant main effect of problem $\left[F(2,24)=37.43, M S_{\mathrm{e}}=2,028.99, p<.01, \eta^{2}=.757\right]$. Post hoc tests using Tukey's HSD method revealed significant differences in time to solve each of the three training problems. Inspection of the means indicated that participants got faster across training problems. The goal specificity $\times$ problem interaction was not significant $\left[F(2,24)<1, \eta^{2}=.061\right]$.

For the graduate participants, there was a significant main effect of problem $\left[F(2,40)=42.17, M S_{\mathrm{e}}=\right.$ $\left.1,633.45, p<.01, \eta^{2}=.678\right]$. Again, post hoc tests using Tukey's HSD method revealed significant differences in time to solve each of the three training problems, confirming that participants got faster with each problem. Neither the main effect of goal specificity nor the goal specificity $\times$ problem interaction was significant $\left[F(1,20)=2.18, M S_{\mathrm{e}}=4,499.25, p>.10, \eta^{2}=\right.$ .098 , and $F(2,40)<1, M S_{\mathrm{e}}=1,633.45, \eta^{2}=.047$, respectively].

Structural knowledge. Relatedness ratings were submitted to the Pathfinder scaling algorithm to generate a 


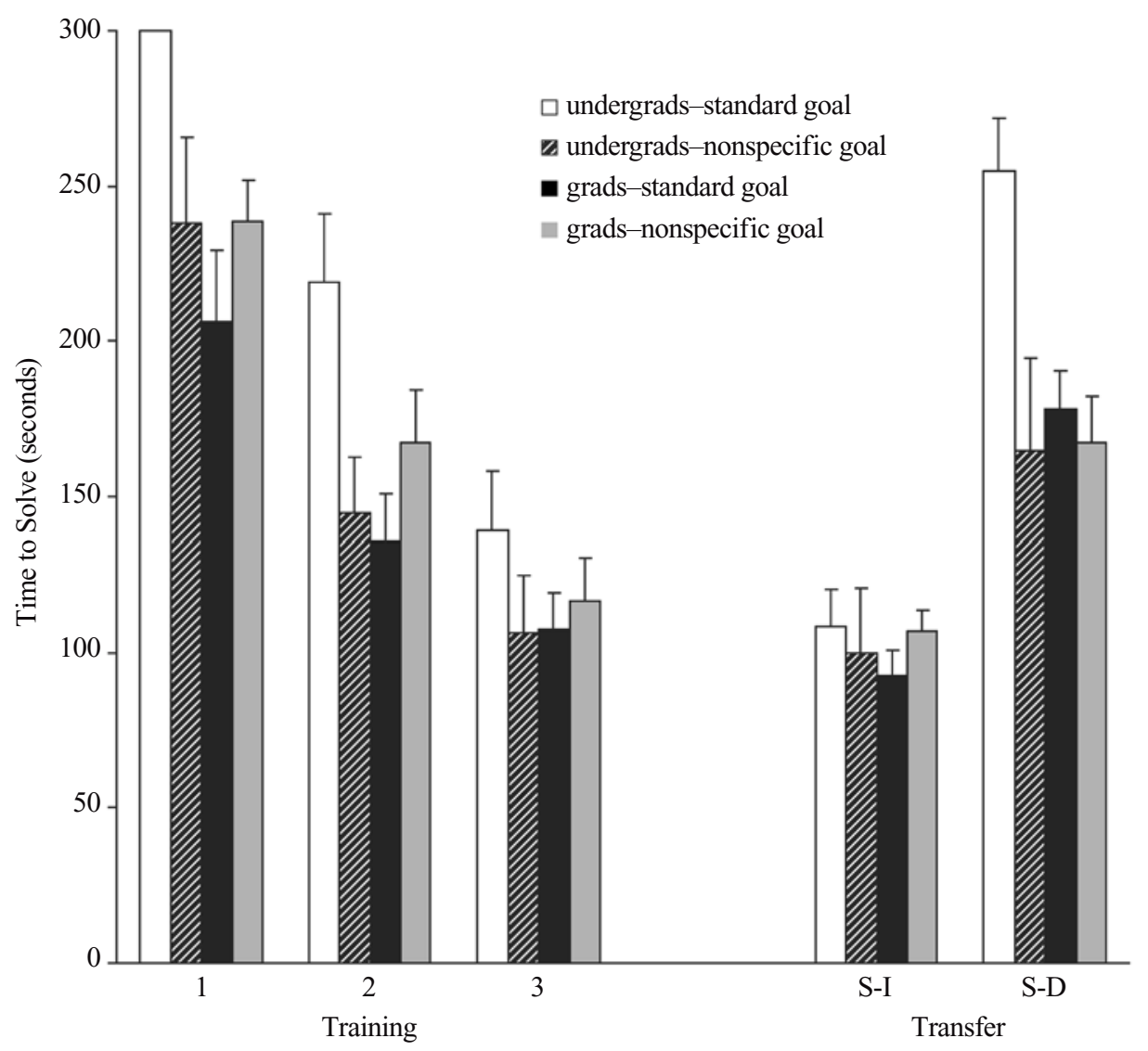

Figure 1. Mean time to solve training and transfer problems as a function of condition.

network representation of each participant's structural knowledge. Parameter values of $r=1, q=\infty$ were used. The $r$ parameter value indicates that relatedness ratings were treated as Euclidian distances, and the $q$ parameter indicates that all possible paths were evaluated in determining whether a direct path between concepts was the shortest and should be included as a link in the network representation. Networks were then analyzed for the number of links to the training goal $\left(S S_{B}\right)$ and the number of links among concepts contained within any given equation on the index cards provided to participants. Because there were a total of six concepts in each network, there were five possible links to the goal $\left(S S_{B}-a, S S_{B}-d f_{B}\right.$, $\left.S S_{B}-M S_{B}, S S_{B}-F, S S_{B}-M S_{W}\right)$. A total of seven links can be inferred from the equations $\left(d f_{B}-a, M S_{B}-S S_{B}, M S_{B}-d f_{B}\right.$, $\left.S S_{B}-d f_{B}, F-M S_{B}, F-M S_{W}, M S_{B}-M S_{W}\right)$. Two links with the goal could also be inferred from the equations $\left(S S_{B}-d f_{B}\right.$, $\left.S S_{B}-M S_{B}\right)$. These two links were thus discarded from the analysis because it was expected that they would be acquired by participants in both the SG and NG conditions. This left three possible links with the goal and five links depicting local relations that could be inferred from the equations for analysis. We refer to the three links with the goal as irrelevant because none was found in a network representation that was derived from the averaged ratings of a pair of statistics experts in a previous study (Trumpower, 2000). Of the five links that could be inferred from the equations, four were present in the expertderived network. The link that was not present in the expert network was discarded from analysis, and the remaining four links are referred to as relevant (it should be noted that analyses were performed both with and without this link included as a relevant link, and similar patterns of results were obtained). On the basis of an attentional focus explanation of the goal specificity effect, we predicted that undergraduate participants in the SG condition would have more irrelevant links with the training goal than would those in the NG condition, whereas undergraduate participants in the NG condition would have more relevant links, depicting the local relations suggested by the equations, than would those in the SG condition. We predicted no goal specificity effects for the graduate participants. However, because of their previous statistics experience, we expected that graduate participants would have a greater proportion of relevant than irrelevant links.

A 2 (goal specificity) $\times 2$ (link type) mixed ANOVA with repeated measures on the second factor was performed on the proportion of possible links present in the networks (see Figure 2). For undergraduate participants, 


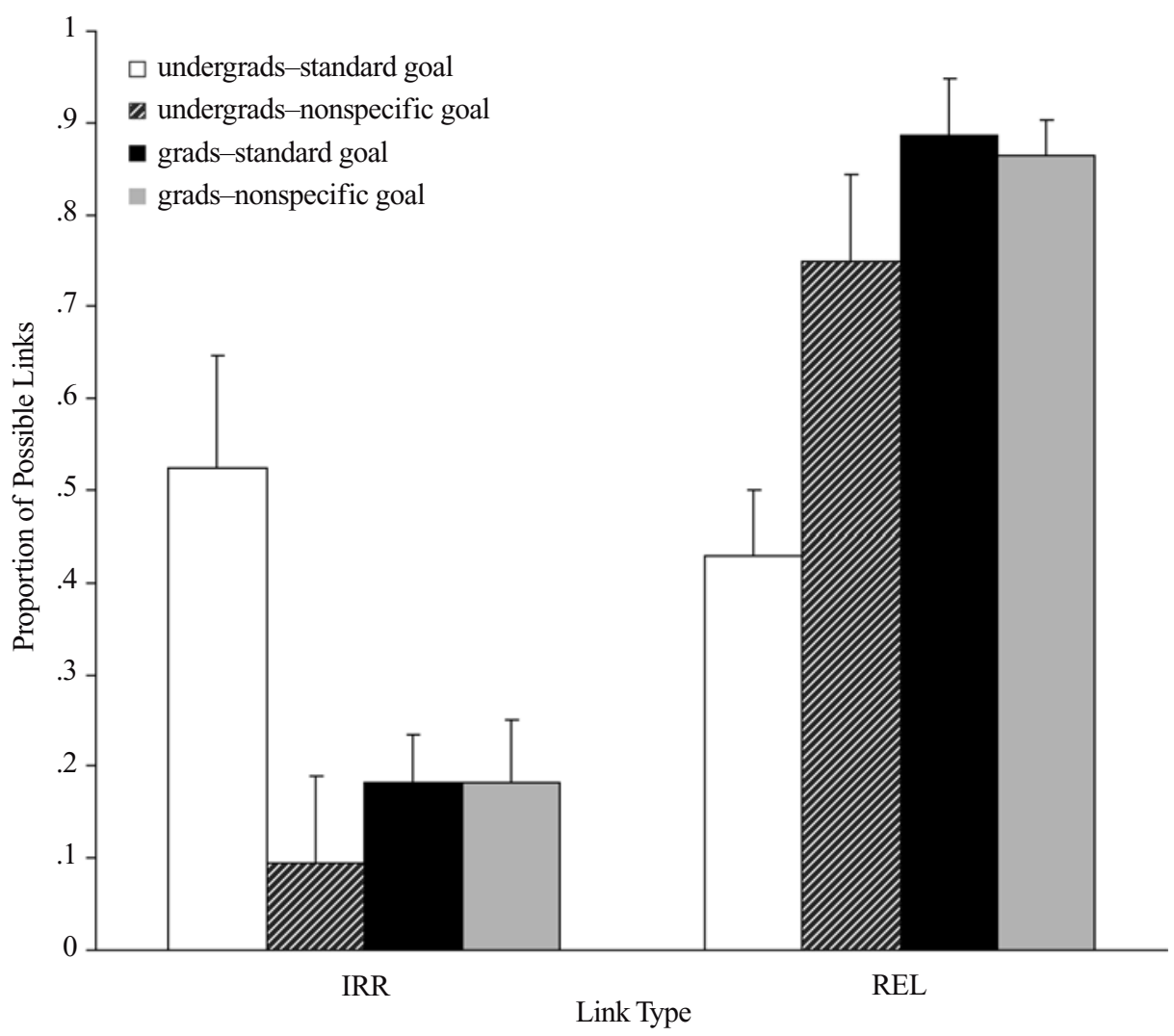

Figure 2. Mean proportion of irrelevant (IRR) and relevant (REL) links acquired as a function of condition.

there was a significant main effect of link type $[F(1,12)=$ $\left.6.87, M S_{\mathrm{e}}=.08, p<.05, \eta^{2}=.364\right]$, which was qualified by a significant goal specificity by link type interaction $\left[F(1,12)=12.34, M S_{\mathrm{e}}=.08, p<.01, \eta^{2}=.507\right]$. The main effect of goal specificity was not significant $\left[F(1,12)<1, M S_{\mathrm{e}}=.05, \eta^{2}=.030\right]$. Simple effects tests confirmed our hypotheses. Participants in the SG condition possessed significantly more irrelevant links than did those in the NG condition $\left[F(1,12)=7.59, M S_{\mathrm{e}}=.08\right.$, $p<.025, \eta^{2}=.387$ ], whereas participants in the NG condition possessed significantly more relevant links than did those in the SG condition $\left[F(1,12)=7.36, M S_{\mathrm{e}}=.05\right.$, $\left.p<.025, \eta^{2}=.380\right]$.

For graduate participants, there was a significant main effect of link type $\left[F(1,20)=133.32, M S_{\mathrm{e}}=.04, p<\right.$ $.01, \eta^{2}=.869$ ], such that they possessed a greater proportion of relevant than irrelevant links. Neither the main effect of goal specificity nor the interaction was significant $\left[F(1,20)<1, M S_{\mathrm{e}}=.03, \eta^{2}=.043\right.$ and $F(1,20)<$ $1, M S_{\mathrm{e}}=.04, \eta^{2}=.027$, respectively]. These results are consistent with the hypotheses that more expert participants should already possess more expert-like knowledge (i.e., relevant links) and that they should not be influenced by the goal specificity manipulation.

Transfer. The average time to solve the S-I and S-D transfer problems was analyzed with a 2 (goal speci- ficity) $\times 2$ (transfer type) mixed ANOVA with repeated measures on the second factor (see Figure 1). For undergraduate participants, there was a significant main effect of transfer type $\left[F(1,12)=46.31, M S_{\mathrm{e}}=1,697.12, p<\right.$ $\left..01, \eta^{2}=.794\right]$ and a marginally significant main effect of goal specificity $\left[F(1,12)=3.84, M S_{\mathrm{e}}=4,436.53, p=\right.$ $\left..07, \eta^{2}=.242\right]$. Both, however, were qualified by a significant goal specificity $\times$ transfer type interaction $\left[F(1,12)=6.83, M S_{\mathrm{e}}=1,697.12, p<.05, \eta^{2}=.363\right]$. Simple effects tests revealed no significant difference between the goal specificity conditions on time to solve S-I transfer problems $\left[F(1,12)<1, M S_{\mathrm{e}}=2,026.86\right.$, $\left.\eta^{2}=.011\right]$, but participants in the NG condition solved S-D transfer problems faster than did those in the SG condition $\left[F(1,12)=6.90, M S_{\mathrm{e}}=4,106.79, p<.025\right.$, $\left.\eta^{2}=.365\right]$.

The difference on S-D transfer problems confirms our hypothesis and extends the generality of the goal specificity effect. The lack of a difference on the S-I transfer problems was unexpected. Previous studies have shown the goal specificity effect using transfer problems that are structurally identical to training problems (Ayres, 1993; Bobis et al., 1994; Sweller, 1988; Sweller et al., 1983; Vollmeyer et al., 1996). It is possible that the problems used in the present study were easier than those used in previous studies. For example, Vollmeyer et al. 
used a more complex, dynamic problem-solving task than that used in the present study. Indeed, all participants in the present study solved the final training problem and both S-I transfer problems correctly in the allotted 5-min time period, and the average time taken to solve each S-I transfer problem was less than $2 \mathrm{~min}$. In contrast, participants in Ayers' study failed to solve for approximately $40 \%$ of the possible angles in their geometry problem-solving task, and Bobis et al.found that $70 \%-80 \%$ of participants could not solve a transfer task after training with a standard goal. Therefore, it seems plausible that our lack of a goal specificity effect on S-I transfer problems may be due to a ceiling effect.

For the graduate participants, we found a significant main effect of transfer type $\left[F(1,20)=53.76, M S_{\mathrm{e}}=\right.$ $\left.1,090.36, p<.01, \eta^{2}=.729\right]$. The graduate participants solved S-I problems faster than they did S-D problems. Neither the main effect of goal specificity nor the interaction was significant $\left[F(1,20)<1, M S_{\mathrm{e}}=1,580.13\right.$, $\eta^{2}=.002$ and $F(1,20)=1.61, M S_{\mathrm{e}}=1,090.36, p>.10$, $\eta^{2}=.074$, respectively]. These results confirmed our hypothesis that the goal specificity effect would not emerge for more experienced problem solvers.

\section{DISCUSSION}

Results of the present study extend prior research on the goal specificity effect. We have shown that NG training can facilitate transfer performance on problems that are structurally different from training problems. We also have shown that the specificity of goals during training influences structural knowledge acquisition in a manner consistent with an attentional focus view. Finally, we have shown that the goal specificity effect is restricted to individuals with low prior domain knowledge.

Previous studies have found that eliminating specific goals during training allows enhanced performance when solving structurally similar transfer problems. Sweller et al. (1983) interpreted this as evidence that NG training facilitates the transition to expertise, and they explained the finding by positing that NG training leads to acquisition of specific schemas based "not on physical principles but rather on a highly specific combination of givens and goal" (Sweller et al., 1983, p. 647). We believe that the hallmark of expertise is not only the ability to solve domain problems efficiently, but the ability to solve novel problems, which requires more general knowledge than the specific schemas posited by Sweller et al. Although our participants were never trained on problems for which the goal was to find $a$ or $M S_{W}$, those in the NG condition solved these structurally different transfer problems faster than did participants in the SG condition. This is consistent with the idea that NG training leads to acquisition of general schemas that are not tied to one particular problem structure. Thus, our finding is congruent with, and as we argued earlier, a more direct test of, Owen and Sweller's (1985) contention that NG training allows acquisition of principles rather than procedures.
Our finding of more general transfer in the NG condition fits nicely with discovering better structural knowledge in the NG condition. Both are well explained by the attentional focus view. According to Miller et al. (1999), predictions regarding transfer should be based on an analysis of where attention is focused and, consequently, what knowledge can be attained during learning, rather than based on a simple analysis of cognitive load. When one is solving SG problems, attention tends to be focused on the goal and, more specifically, on differences between the current problem state and the goal. By focusing attention on relations between the current states and the goal, SG problems divert attention from the relationships between the current state and the next attainable state (i.e., local relations). When one is solving NG problems, attention is focused on getting from the current state to the next attainable state, rather than achieving some distant goal state. Thus, NG problems promote learning of local relations.

These predictions were borne out in our structural knowledge assessment. Participants in the SG condition learned more relations with the goal than did participants in the NG condition, but fewer local relations that did not involve the goal. Whereas the structural knowledge associations obtained in the SG condition were predominantly with a single-goal concept, those obtained in the NG condition involved local relations among a variety of concepts. In this sense, the structural knowledge obtained by SG participants was narrower, and that obtained by NG participants was more general. Thus, when SG participants were given an S-D transfer problem that had a different goal than the training problems, they had little structural knowledge involving the new goal to aid their solution. NG participants did possess useful knowledge, in that they were more likely to know, for instance, the local relations among $F, M S_{B}$, and $M S_{W}$. If asked to solve for $M S_{W}$, for example, NG participants possessed more relevant knowledge involving $M S_{W}$ than did SG participants. Differential structural knowledge acquired by NG and SG participants may account for the difference between the conditions in time needed to solve S-D transfer problems.

Although our results are consistent with an attentional focus explanation, they do not rule out other explanations. For example, Vollmeyer and colleagues (Vollmeyer et al., 1996; Burns \& Vollmeyer, 2002) have proposed a dual-space explanation for the goal specificity effect. They contend that NG problems encourage search through rule space, whereas SG problems encourage search through instance space. Rule space contains the rules that govern a problem-solving task, whereas instance space is comprised of all possible states, or instances, of a task. According to this account, the goal specificity effect arises because search in rule space facilitates rule induction, whereas search in instance space does not. To the extent that attending to local relations is analogous to searching rule space, that attending to a specific goal is analogous to searching instance space, and that the rules induced as a result of searching rule 
space resemble the general knowledge acquired by NG participants in the present study, the attentional focus and dual-space accounts are compatible explanations of the goal specificity effect.

The attentional focus perspective is also compatible with a version of CLT that includes both extraneous and germane cognitive load. Attentional focus may be thought of as the allocation of cognitive resources. From this perspective, germane cognitive load can be defined as the result of attending to (and resources being allocated to the processing of) relevant relations, whereas extraneous cognitive load can be defined as the result of attending to (and resources being allocated to the processing of) irrelevant relations. Distinguishing relevant from irrelevant relations may be determined empirically, and/or through task analysis. In the present study, relevant relations were deemed relevant on the basis of their applicability to both S-I and S-D transfer problems, whereas irrelevant relations were deemed irrelevant based on their more limited applicability to S-I transfer problems only. This distinction was verified by empirically derived structural knowledge representations of statistics experts (Trumpower, 2000). Experts possessed links corresponding to relevant relations, but not to irrelevant relations. Thus, the structural knowledge measure used in the present study can be viewed as a (heretofore nonexistent) measure of germane and extraneous load and thus represents an important development for cognitive load research.

Implications of the present study are in line with those of previous studies of the goal specificity effect: Problems used during initial training should have nonspecific goals. Or, according to the attentional focus view, problems used during initial training should focus attention on local relations. This includes problems with nonspecific goals but could include any pedagogically targeted problems. We stress that this applies to initial training. Our results extend previous findings by showing that the goal specificity effect only applies to problem solvers with little prior domain knowledge. After one has gained a certain amount of experience, NG training appears to lose its benefit, as the knowledge acquired via NG training, which facilitates transfer to novel problems, is already possessed by the domain experts.

\section{REFERENCES}

AYres, P. L. (1993). Why goal-free problems can facilitate learning. Contemporary Educational Psychology, 12, 376-381.

BJORK, R. A. (1999). Assessing our own competence: Heuristics and illusions. In D. Gopher \& A. Koriat (Eds.), Attention and performance XVII: Cognitive regulation of performance. Interaction of theory and application (pp. 435-459). Cambridge, MA: MIT Press.

Bobis, J., Sweller, J., \& Cooper, M. (1994). Demands imposed on primary-school students by geometric models. Contemporary Educational Psychology, 19, 108-117.

BURNS, B. D., \& VOLLMEYER, R. (2002). Goal specificity effects on hypothesis testing in problem solving. Quarterly Journal of Experimental Psychology, 55A, 241-261.

Chi, M. T. H., Feltovich, P. J., \& Glaser, R. (1981). Categorization and representation of physics problems by experts and novices. $C o g-$ nitive Science, 5, 121-152.

GeDDes, B., \& STEVENSON, R. (1997). Explicit learning of a dynamic system with a non-salient pattern. Quarterly Journal of Experimental Psychology, 50A, 742-765.

GoldSMith, T. E., JOHNSON, P. J., \& Acton, W. H. (1991). Assessing structural knowledge. Journal of Educational Psychology, 83, 88-96.

Kalyuga, S., Chandler, P., \& Sweller, J. (2000). Incorporating learner experience into the design of multimedia instruction. Journal of Educational Psychology, 92, 126-136.

Larkin, J. H., McDermott, J., Simon, D. P., \& Simon, H. A. (1980). Expert and novice performance in solving physics problems. Science, 208, 1335-1342.

Miller, C. S., Lehman, J. F., \& Koedinger, K. R. (1999). Goals and learning in microworlds. Cognitive Science, 23, 305-336.

Owen, E., \& Sweller, J. (1985). What do students learn while solving mathematics problems? Journal of Educational Psychology, 77, 272284.

RenkL, A. (2002). Learning from worked-out examples via selfexplanations: How it can(not) be fostered. In Proceedings of the Twenty-Fourth Annual Conference of the Cognitive Science Society (p. 49). Mahwah, NJ: Erlbaum.

ScHROTH, M. L. (1997). The effects of different training conditions on transfer in concept formation. Journal of General Psychology, 124, 157-165.

SchVaneveldt, R. W. (1990). Pathfinder associative networks: Studies in knowledge organization. Norwood, NJ: Ablex.

Simon, D., \& Simon, H. (1978). Individual differences in solving physics problems. In R. Siegler (Ed.), Children's thinking: What develops? Hillsdale, NJ: Erlbaum.

Sweller, J. (1988). Cognitive load during problem solving: Effects on learning. Cognitive Science, 12, 257-285.

Sweller, J., \& CoOPER, G. A. (1985). The use of worked examples as a substitute for problem solving in learning algebra. Cognition \& Instruction, 2, 59-89.

SWELlER, J., \& LEVINe, M. (1982). Effects of goal specificity on means-ends analysis and learning. Journal of Experimental Psychology: Learning, Memory, \& Cognition, 8, 463-474.

Sweller, J., MaWer, R. F., \& Ward, M. R. (1983). Development of expertise in mathematical problem solving. Journal of Experimental Psychology: General, 112, 639-661.

TRUMPOWER, D. L. (2000). Schema acquisition and solution strategy in statistics problem solving. In Proceedings of the Twenty-Second Annual Conference of the Cognitive Science Society (p. 1061). Mahwah, NJ: Erlbaum.

TuOvinen, J. E., \& Sweller, J. (1999). A comparison of cognitive load associated with discovery learning and worked examples. Journal of Educational Psychology, 91, 334-341.

VALCKE, M. (2002). Cognitive load: Updating the theory? Learning \& Instruction, 12, 147-154.

van Merriënboer, J. J. G., Schuurman, J. G., De Croock, M. B. M., \& PaAs, F. G. W. C. (2002). Redirecting learners' attention during training: Effects on cognitive load, transfer test performance and training efficiency. Learning \& Instruction, 12, 11-37.

Vollmeyer, R., Burns, B. D., \& Holyoak, K. J. (1996). The impact of goal specificity on strategy use and the acquisition of problem structure. Cognitive Science, 20, 75-100. 


\section{APPENDIX \\ Example Training and Transfer Problems}

\section{Training Problem}

Students in a Psych 106L class decide to carry out an experiment to determine the effects of listening to music while studying on text comprehension. They find a mean square within of 35.01 and an $F$ ratio of 1.42. The 5 groups used in the experiment were as follows: Group 1 listened to rock music, Group 2 listened to classical music, Group 3 listened to bluegrass music, Group 4 listened to rap music, and Group 5 listened to no music. What was the sum of squares between for their experiment?

\section{Transfer Problem-Structurally Identical}

A study reported in the Journal of Clinical Psychology compared the effectiveness of 3 different psychotherapeutic methods for the treatment of severe depression. In the results section, the researchers report a mean square within of 102.10 and an $F$ ratio of 3.96. What was the sum of squares between?

\section{Transfer Problem-Structurally Different}

A cognitive psychologist is interested in the effects of presentation modality on memory for word lists. She divides subjects into 3 groups: One group is presented with a word list visually on a computer screen, a second group is presented the same word list verbally through headphones, and the third group is presented the list tactilely with Braille writing. Later, all 3 groups are asked to recall as many words from the list as possible. A sum of squares between of 77.10 and an $F$ ratio of 1.50 are obtained. What is the mean square within for this study?

(Manuscript received March 12, 2003;

revision accepted for publication April 9, 2004.) 